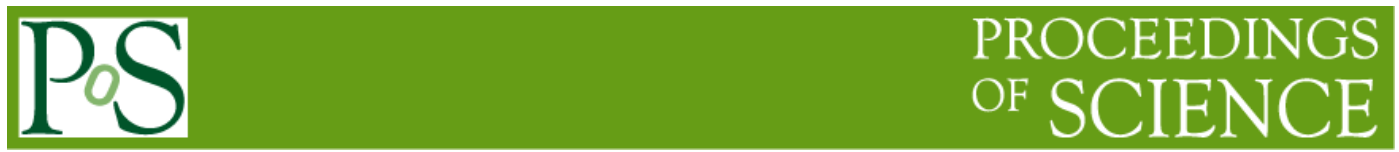

\title{
Polarized beams in AGS and RHIC in Run-2015
}

\author{
A. Zelenski ${ }^{1}$ \\ $B N L$ \\ Upton, USA \\ E-mail: zelenski@bnl.gov
}

\begin{abstract}
The upgraded high-intensity polarized source operation and improvements in the injector chain and at RHIC have allowed higher bunch intensities and lower emittance for colliding beams in RHIC. The successful commissioning of the electron lenses resulted in operation at a higher beam-beam parameter and therefore higher luminosity. Polarization values in collisions $60-65 \%$ were achieved at high luminosity operation at beam energies of a $\sqrt{S}=200 \mathrm{GeV}$. For the first time polarized proton-Gold, proton-Aluminum collisions were studied.
\end{abstract}

XVI International Workshop on Polarized Sources, Targets, and Polarimetry

September 14-18, 2015

Bochum, Germany

1

Speaker 


\section{Introduction}

The goals of the first ten weeks with colliding polarized proton beams of $100 \mathrm{GeV}$ each were studies of the gluon contribution to the proton spin with longitudinally polarized beams and of the transversity (Sivers effect) with transversely polarized colliding beams.

Due to higher bunch intensity integrated beam luminosity in Run-2015 exceeded the luminosity in all combined. For the first time polarized proton-gold, proton-aluminum collisions with high luminosity and polarization were obtained in RHIC for saturation-physics studies and as a reference for heavy ion collisions.

The largest effort was commissioning of the electron lenses, one in each ring, which are designed to compensate the effect of one of the two beam-beam interactions experienced on the proton bunches. The e-lenses increase the bunch intensity above which the luminosity becomes limited by the beam-beam interaction. In previous runs, strong space-charge forces at injection to the Booster caused the emittance growth and did not allow to take full benefits of the high polarized source and Linac pulse intensity. In Run 2015, a different capture scheme was implemented, which defocuses the center of the bunch longitudinally and reduces the peak current. The resulting transverse emittances at the AGS extraction and in RHIC were 20\% smaller than in previous runs primarily due owing to this improvement. The higher brightness the Booster and AGS beam allowed to take advantage of the increased limit on the maximum bunch intensity and resulted in significant increase of the integrated beam luminosity. As a result, the total integrated luminosity in Run 2015 exceeded the integrated luminosity of all previous runs (see Figure 1) [1].

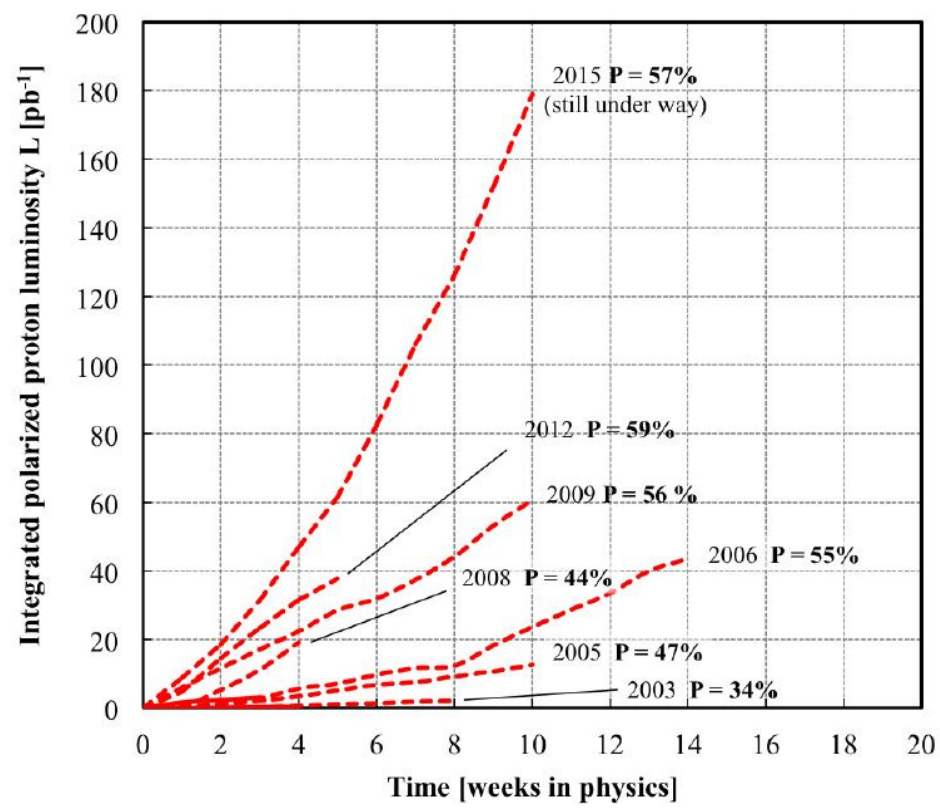

Figure1. RHIC luminosity for pp collision in the Runs 2003 to 2015. 


\section{Polarized source upgrade}

A novel polarization technique had been successfully implemented for the RHIC polarized $\mathrm{H}^{-}$ion source upgrade to higher intensity and polarization. In this technique a proton beam inside the high magnetic solenoid field is produced by ionization of the atomic hydrogen beam (from external source) in the He ionizer cell. Proton polarization is produced by capture of polarized electron from the optically-pumped $\mathrm{Rb}$ vapor. The use of the high-brightness primary beam and the charge-exchange cross-sections resulted in production of high intensity $\mathrm{H}^{-}$ion beam of $85 \%$ polarization [2]. The source very reliably delivered polarized beams in the RHIC Run-2013 and Run-2015. High values of beam current, brightness and polarization resulted in $70-74 \%$ polarization at $23 \mathrm{GeV}$.

The polarized source OPPIS at RHIC, based on an ECR primary proton source was retired after successful operation in Runs 2000 to 2012. The new source with the atomic hydrogen beam injector and the He-ionizer was developed in 2010 to 2012 and commissioned for operation in Run-2013. It produces significantly higher brightness of the primary proton beam, which resulted in higher intensity and polarization of the beam delivered for injection to Linac-Booster-AGS-RHIC accelerator chain. Practically all OPPIS systems were modified (in addition to the ECR-source): a new superconducting solenoid; a new He-ionizer cell with a pulsed He-injection and new pulsed electro-dynamic gas valve; the beam energy separation system developed for un-polarized residual beam suppression; the new vacuum system with turbo-molecular pumps for He-pumping; the new laser control, diagnostics and transport systems. The Low Energy Beam Transport system (LEBT) was modified to reduce losses of the high-intensity beam and to increase the energy resolution for better suppression of the residual unpolarized beam component. The performance of the new source and the beams in Run 2015 is presented in Table 1.

Table 1. Characteristic values of the Booster injection system in the Run 2015. The Linac pulse length is $300 \mu \mathrm{s}$.

\begin{tabular}{|l|c|c|c|l|}
\hline $\begin{array}{l}\text { Rb-cell thickness }\left[\mathrm{NL} \times 10^{13}\right. \\
\left.\text { atoms } / \mathrm{cm}^{2}\right]\end{array}$ & 4.5 & 5.5 & 7.5 & 10.4 \\
\hline Linac current, $\boldsymbol{\mu A}$ & 500 & 560 & 680 & 750 \\
\hline Booster input, $\times 10^{11}[$ ions/pulse $]$ & 9.0 & 10.0 & 12.2 & 13.5 \\
\hline Polarization at $200 \mathrm{MeV}, \%$ & 84.0 & 83.0 & 80.5 & 78.5 \\
\hline
\end{tabular}

Very reliable operation and reduced maintenance time was achieved. Already in the first year of operation in Run - 2013, the new source performance exceeded the old ECR-based source parameters and performances were further improved in Runs 2014 and 2015. The high 
values of beam current, brightness and polarization out of the source and the Linac resulted in $75 \%$ polarization at $23 \mathrm{GeV}$ out of the AGS (due to beam emittance reduction by strong beam scraping at extraction from the Booster) and in 60 to $65 \%$ polarization of the colliding beams in RHIC at beam energies of a 100 to $250 \mathrm{GeV}$.

\section{Polarization}

In the RHIC complex, there are two absolute proton polarimeters: the elastic protoncarbon polarimeter at beam energy $200 \mathrm{MeV}$ and the CNI (Coulomb Nuclear Interference) proton-proton $\mathrm{H}$-jet polarimeter at the RHIC store energies of $23-255 \mathrm{GeV}$ [3]. The polarization of the $200 \mathrm{MeV}$ beam is $80-84 \%$ and was measured with an accuracy of better than $0.5 \%$. The $\mathrm{H}$-jet polarimeter measures the average polarization over the whole beam intensity profile. The polarization transport simulations show that depolarization occurs at the edge of the beam and polarization of the beam core (center of the beam intensity profile) should be preserved during acceleration [4]. The polarization distribution across the beam intensity profile (beam polarization profile) is measured by the scanning p-Carbon CNI polarimeters in AGS and RHIC [5]. These measurements produce complimentary information to the $\mathrm{H}$-jet polarimeter on polarization losses along the accelerator chain. The knowledge of polarization profiles of both beams are also required for calculation of the polarization values for colliding beams, which are used for the normalization of experimental spin asymmetries.

The polarization profiles at $200 \mathrm{MeV}$ after the Linac and after the Booster at injection to AGS are flat, which is consistent with no losses in Booster after the careful tuning of the orbit harmonics. In the AGS the use of two partial helical snakes and jump quadrupoles greatly improved the polarization preservation, but still there are some losses depending on beam emittance. The non-flat polarization profiles at AGS flattop energy were measured by the CNI polarimeters in AGS and at injection to RHIC. The AGS polarization versus beam intensity is presented in Figure 2. Polarization in excess of $70 \%$ was achieved at beam bunch intensities up to $2.4 \cdot 10^{11}$ bunch. Extrapolation to zero bunch intensity gives polarization value about $80 \%$, which is close the polarization value measured in $200 \mathrm{MeV}$ polarimeter as expected from a polarization loss model. Polarization profiles were also measured at different beam intensities and are also consistent with the polarization loss model.

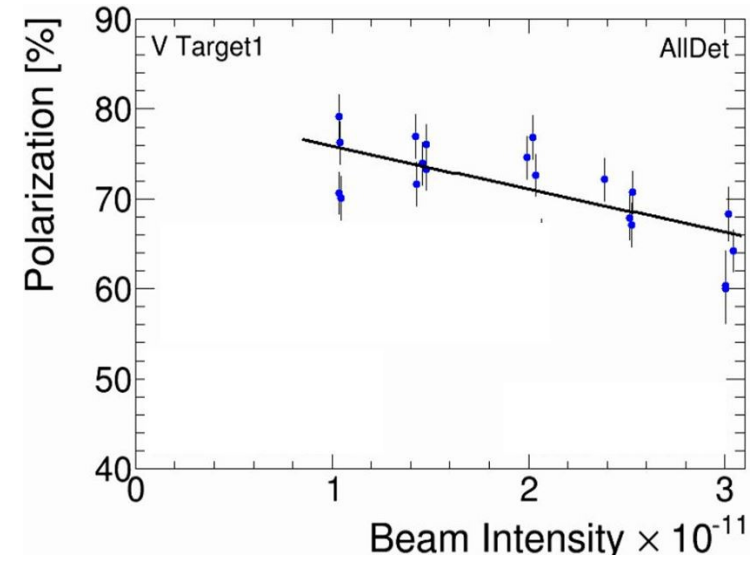

Figure 2. AGS polarizations dependence vs. bunch intensity. 
The knowledge of absolute polarization measurements at different beam energies is very important for understanding of the polarization losses during acceleration and transport in the RHIC accelerator chain: Source-Linac-Booster-AGS-RHIC. The combination of polarization transport simulation in AGS and RHIC and absolute polarization measurements tools at 200 $\mathrm{MeV}$ and RHIC store energies provide complimentary information on polarization losses along the RHIC accelerator chain.

A number of major upgrades were completed for Run-2015 to improve H-jet performances and reliability: a new high-purity hydrogen gas generator (palladium based) and a new set of RF transition power supplies. As a result, the $\mathrm{H}$-jet operation at high beam intensity increased to two weeks between the maintenances.

Limitations of $\mathrm{p}$-Carbon polarimeters operation at high beam intensity increased the value of $\mathrm{H}$-jet polarimeter measurements. New Si-strip detectors, developed for Run-2015, reduced statistical errors by about 1.5-2.0 times due to larger solid angle and extended kinematic range. The extended kinematic range also provided better handle on systematic errors contributions. Data acquisition upgrade with new Wave Form Digitizers (WFD) took advantage of the higher energy resolution and improved the background discrimination.

Figure 3 shows the measured H-jet polarization for all RHIC fills in Run-2015. The Yellow ring (in the Yellow ring beam is moving counter-clockwise) polarization was 2-3\% higher than in Blue ring (in the Blue ring beam is moving clockwise) as expected from polarization direction mismatch in between AGS and RHIC rings. In addition, the stronger polarization decay was observed in the Blue ring, in particular at the end of the Run. Intermittent polarization losses, observed in AGS at high beam intensity may be caused by instabilities in the jump- quadrupole timing.

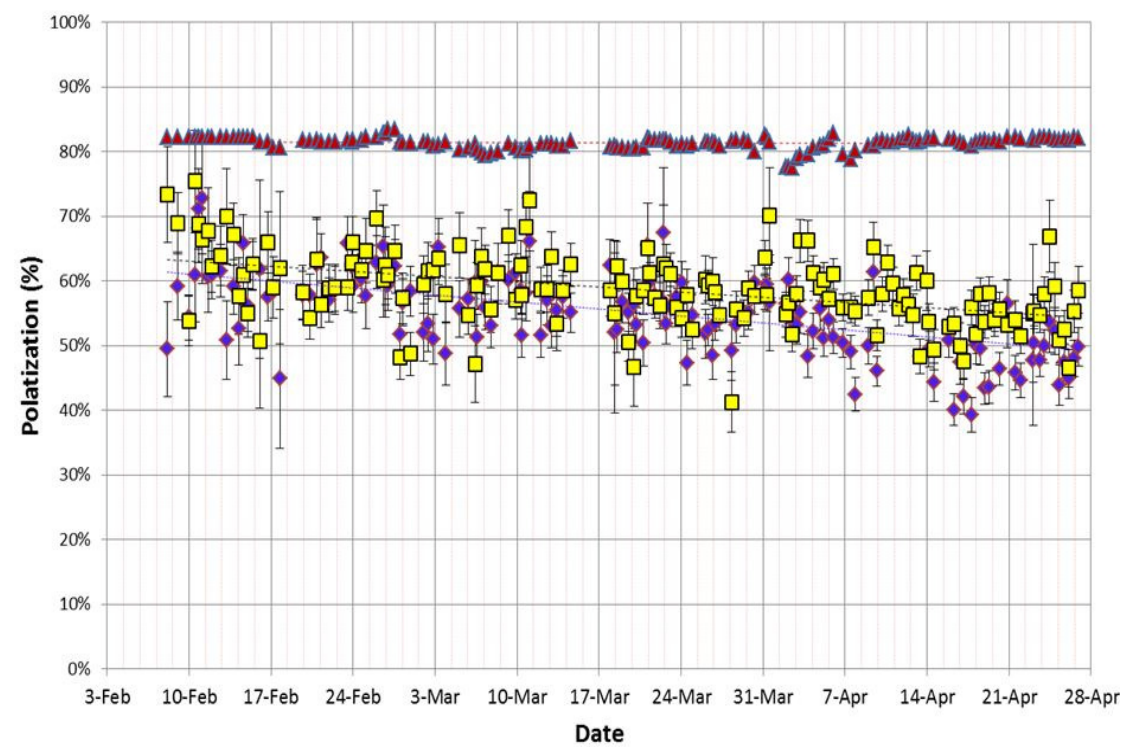

Figure 3. H-jet polarization measurements for pp-collisions in Run-2015 (yellow squaresmeasuruments in Yellow ring; magenta diamonds- measurements in Blue ring; red trianglesmeasurements at $200 \mathrm{MeV}$ ). 


\section{Summary}

The high-intensity polarized source and improvements in the injector chain and RHIC have allowed operation at higher bunch intensities and lower emittance. The successful commissioning of the electron lenses has allowed to take advantage of higher bunch intensities due to reduction of the beam-beam interaction. As a result, the integrated luminosity for the Run -2015 exceeded the combined luminosity delivered in Runs 2003 to 2013. For the first time polarized proton-Gold, proton-Aluminum collisions were studied. Still there are polarization losses of 10 to $15 \%$ in the AGS (intensity dependence, instabilities) and of 5 to $10 \%$ in RHIC (polarization decay, larger with spin-rotators). Polarization of 60 to $65 \%$ in the collisions experiments was achieved in high luminosity operation at: $\sqrt{ } \mathrm{S}=200 \mathrm{GeV}$.

\section{Acknowledgements}

The author acknowledges contributions by G. Atoian, J. Ritter, Y. Makdisi, H. Huang, V. Schoefer and many other RHIC staff to the source and polarimeter operation and by A. Poblaguev in the data analysis. This work was supported by BSA, LLC under Contract No. DEAC02-98CH10886 with the U.S. DOE.

\section{References}

[1] V. Schoefer et al., Proc. of IPAC2015, TUPW1060, 2015.

[2] A. Zelenski et al., Proc of ICIS 2015, Rev. Sci. Instr., 87, 02B705, 2015.

[3] A. Zelenski et al., NIM, A536, p.248, 2005.

[4] A. Zelenski et al., PoS (PSTP 2013) 056, 2013.

[5] I. Nakagava et al., AIP Conf. Proc., 980, p.380, 2008. 